\title{
Comparison of 3D printed anatomical model qualities in acetabular fracture representation
}

\author{
David A. Salazar ${ }^{\wedge}$, Justin Cramer ${ }^{2}$, Nicholas W. Markin ${ }^{3}$, Nathaniel H. Hunt ${ }^{1}$, Gabe Linke ${ }^{4}$, \\ Justin Siebler ${ }^{5}$, Jorge Zuniga ${ }^{1}$ \\ ${ }^{1}$ Department of Biomechanics, University of Nebraska Omaha, Omaha, NE, USA; ${ }^{2}$ Department of Radiology, University of Nebraska Medical \\ Center, Omaha, NE, USA; ${ }^{3}$ Department of Anesthesiology, University of Nebraska Medical Center, Omaha, NE, USA; ${ }^{4}$ Department of Radiology, \\ Children's Hospital and Medical Center, Omaha, NE, USA; ${ }^{5}$ Department of Orthopedic Surgery and Rehabilitation, University of Nebraska Medical \\ Center, Omaha, NE, USA \\ Contributions: (I) Conception and design: DA Salazar, J Cramer, NW Markin, NH Hunt, J Zuniga; (II) Administrative support: J Cramer, NW \\ Markin, NH Hunt, G Linke, J Zuniga; (III) Provision of study materials or patients: DA Salazar, J Cramer, NW Markin, G Linke, J Siebler; (IV) \\ Collection and assembly of data: DA Salazar, J Cramer, G Linke; (V) Data analysis and interpretation: DA Salazar, J Cramer, NW Markin, NH \\ Hunt, J Zuniga; (VI) Manuscript writing: All authors; (VII) Final approval of manuscript: All authors. \\ Correspondence to: David A. Salazar. 6160 University Dr S, Omaha, NE 68182, USA. Email: dsalazar@unomaha.edu.
}

Background: Acetabular fractures account for $10 \%$ of pelvis injuries, which are especially difficult to treat in developing countries with less access to resources. 3D printing has previously been shown to be a beneficial method of surgical planning, however the steep initial costs associated with purchasing a 3D printer may prevent some facilities form utilizing this technique. The purpose of this study was to develop 3D printed models for acetabular surgery using methodologies of varying cost to determine differences in model accuracy and overall quality.

Methods: Five acetabular fracture models were developed from de-identified CT data using (I) proprietary and open-source segmentation software and (II) fused deposition modeling (FDM) and stereolithography (SLA) 3D printing methods. The distance between the posterior inferior iliac spine (PIIS) and the ischial spine as well as a unique fracture fragment for each model was compared between the different printing methodologies. The models were then given to 5 physicians and assessed on their overall accuracy compared to traditional 2D images.

Results: Printing methodology did not affect the distance from PIIS to ischial spine $(\mathrm{P}=0.263)$. However, fracture fragment representation differed across $3 \mathrm{D}$ printed models, with the most accurate model produced by the high-end resin-based printer $(\mathrm{P}=0.007)$. The survey analysis showed that the low-cost printing methods produced models that were not as accurate in their representation of the fractured region $(\mathrm{P}=0.008)$. Conclusions: The differences between models developed using traditional methods and low-cost methods have slight differences but may still provide useful information when developing a surgical plan.

Keywords: 3D printing; additive manufacturing; acetabular fracture; anatomic model; surgical planning

Submitted Sep 24, 2021. Accepted for publication Jan 10, 2022.

doi: $10.21037 / \mathrm{atm}-21-5069$

View this article at: https://dx.doi.org/10.21037/atm-21-5069

^ ORCID: 0000-0003-2888-1658. 


\section{Introduction}

Acetabular fracture is defined as damage to the pelvic bone at the hip joint, and accounts for roughly $10 \%$ of the 300,000 hip fractures that occur in the US every year; with an average 37 per 100,000 of these causing damage to the acetabulum as well $(1,2)$. Repair of the pelvic bone can require complex orthopedic intervention at the acetabulum and on occasion the femur as well. These types of acetabular injuries are often caused by one of two issues: high-impact incidents such as falls from large heights or vehicle accidents, or low-impact incidents alongside bone degradation due to age or other comorbidity. The necessity for surgical intervention has been most commonly used in high-impact incidents (such as motor incidents), which have a higher occurrence in developing countries due to things such as industrialization, less access to healthcare, and an increased number of motor vehicles per capita $(2,3)$.

In regard to acetabular repair, less developed countries with limited resources face a number of barriers. The incidence of high-impact fractures is much higher and continuing to increase compared to more developed countries, which may be a result of industrialization (2). Additionally, the number of facilities with trained individuals is significantly less than those in developed countries. Previous studies have reported that while physicians in developing countries are generally trained in orthopedics, a majority of them have never performed an acetabular fracture repair (4). While the outcomes of many of lower-limb orthopedic intra-articular operations are defined as satisfactory, a reported $33 \%$ of acetabular repairs are defined as "fair or poor" and require either follow up visits or additional operations (5). Additional needs for these countries identified by Whiting et al. included things such as resources, equipment, and post-operation therapy to help improve the outcomes of acetabular surgery (4).

One such method that has been used to improve the outcome of surgical interventions is the usage of $3 \mathrm{D}$ printed anatomical models. These models have been used to assist with complex operations ranging from tumor resection within the heart and spine $(6,7)$, and have proven to be especially useful in the field of orthopedics $(8,9)$. Previous studies have found improvements in surgical planning due to increased spatial understanding of the region of interest when using a 3D printed anatomical model (10). This could help to improve surgical outcomes at institutions with less resources, including developing nations. However, many of the models developed for these purposes utilize high-end printers and materials that are not accessible to facilities with less resources. While some models for surgical planning have been developed using low-cost equipment, their accuracy may be of question. Some reports have found significant differences between models developed using different methods (11), which may discourage some hospitals from using 3D printed anatomical models as a part of their surgical planning despite its reported benefits for surgical planning.

The purpose of this study was to develop anatomical models specifically for acetabular repair using previously described methods, as well as low-cost alternatives and to compare the accuracy of the different development methods. The researchers hypothesized that there would be no significant difference between the development methods and that any method of anatomic model development would be beneficial for surgical planning. We present the following article in accordance with the MDAR reporting checklist (available at https://atm.amegroups.com/article/ view/10.21037/atm-21-5069/rc).

\section{Methods}

This study was approved through the IRB at the University of Nebraska Medical Center (258-18-EP) with waiver of consent due to the data being deidentified and accessed retrospectively. Five acetabular fractures, including the entire pelvis, were modeled using two approaches and printed on four 3D printers (total of 20 models). Models were created from the same CT scan using two different segmentation software packages and then $3 \mathrm{D}$ printed on a variety of "high-end" and "low-cost" printers to assess differences between the production methods (Figure 1). The study was conducted in accordance with the Declaration of Helsinki (as revised in 2013).

\section{Experimental procedure}

To assess the printed models' accuracy in replicating the correct size of the patient's pelvic bone, the distance from the posterior inferior iliac spine to the ischial spine was recorded from the $\mathrm{CT}$ scan and compared to physical measurements taken directly on the $3 \mathrm{D}$ printed models. A unique fracture fragment was also measured directly on the CT imaging data and then compared to physical measurements taken from the printed models. To assess the overall quality of the $3 \mathrm{D}$ printed models, they were given to 5 practicing physicians (MD) who completed a survey 


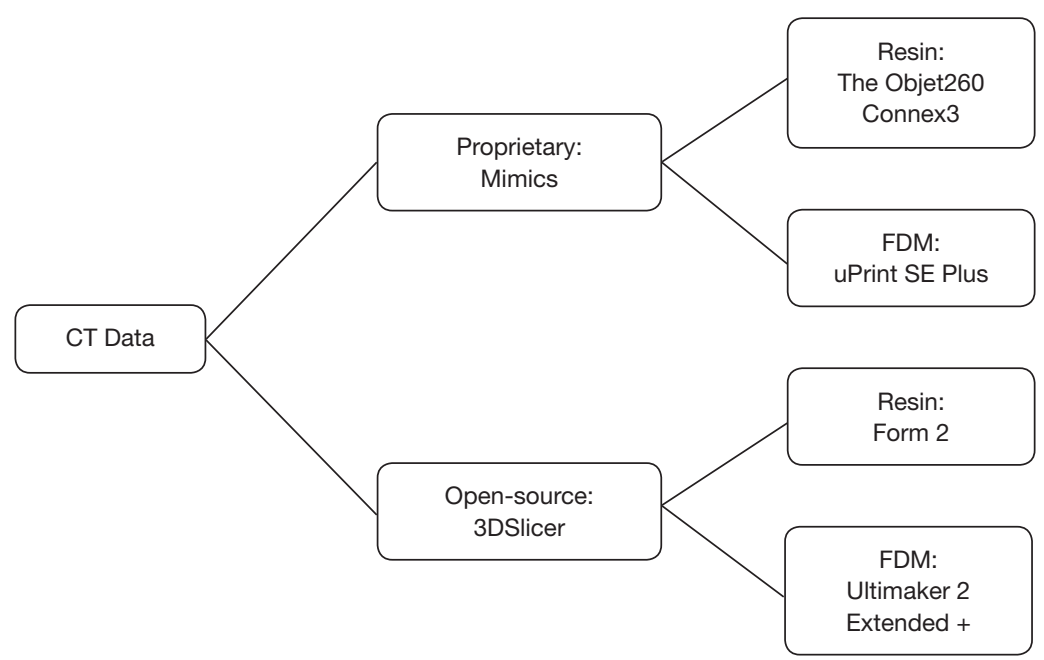

Figure 1 Depiction of the workflow used to produce the anatomical models. High-end models were segmented using the proprietary software Mimics (license cost: \$12,975.00) and were printed on the Objet260 (printer cost: \$158,900.00 and the uPrint (printer cost: $\$ 26,934.00)$. Low-cost models were segmented using the open-source software 3DSlicer (license cost: $\$ 0$ ) and were printed on the Form 2 (printer cost: \$3,350.00) and the Ultimaker 2 Extended+ (printer cost: \$2,999.00). FDM, fused deposition modeling.

for each model. The survey data was analyzed to observe differences in representation and their potential utility within the operating room.

\section{Model development}

The CT scans utilized were acquired from five CT pelvis studies, with slice thicknesses of $1.25 \mathrm{~mm}$ for 4 models and $0.625 \mathrm{~mm}$ for 1 model. In-plane pixel resolution ranged from $0.6-0.8 \mathrm{~mm}$. Models were segmented using semiautomatic thresholding techniques on both Mimics (Version 20.0, Materialise, Belgium; cost \$12,975.00) and 3D Slicer (Version 4.8.1, Brigham and Women's Hospital, Boston, MA; cost free, open-source) (12-14). Segmentation was performed using a combination of semi-automatic and manual thresholding techniques by experienced medical image segmentation specialists and verified by a licensed radiologist. Each segmentation on both 3D Slicer and Mimics took a similar amount of time, at approximately 60 minutes per model. To standardize the orientation of each of the printing methods, all models were printed with the acetabulum facing upward on the build platform. Postprocessing procedures varied per printer: Objet model support was removed via a high-pressure water cleaner (Powerblast, Balco, UK), uPrint support was removed via a dissolvable support solution (ABS, Stratasys, Inc. Eden Praire, MN, USA), Form 2 support was removed manually and soaked in an isopropyl alcohol solvent bath, and Ultimaker support was removed manually.

\section{Model grouping}

Models were developed using two different methods: a high-cost method that involved proprietary segmentation software and high-quality $3 \mathrm{D}$ printers, and a low-cost method that utilized open-source segmentation software and low-cost desktop 3D printers to account for the varying level of resources that different institutions may have. The models segmented using the proprietary software Mimics were printed on two high-end printers and focused on two popular methods of printing: resin-based SLA printing and hard-plastic filament based FDM printing. The printers used in this study include the Objet260 Connex3 (Stratasys, Inc. Eden Prairie, MN; cost $\$ 158,900.00$ ) which is a highend $3 \mathrm{D}$ resin-based printer that is capable of printing with multiple colors and materials, and the uPrint SE Plus (Stratasys, Inc. Eden Prairie, MN; cost \$26,934.00), a high-end industrial FDM printer with dissolvable support structures. The models were also segmented using opensource software 3DSlicer and were printed on two low-cost desktop 3D printers: the Form 2 (Formlabs, Somerville, MA; cost $\$ 3,350.00$ ), a stereolithography (SLA) printer that uses liquid resin, and the Ultimaker 2 Extended + (Ultimaker B.V., Geldermalsen, The Netherlands; cost \$2,999.00), an 
Table 1 Printing and measurement outcomes for each of the respective methods used

\begin{tabular}{lcccc}
\hline Printer & Printing time $(\mathrm{hh}: \mathrm{mm})$ & Material cost $(\$)$ & Measurement 1 accuracy error $(\mathrm{mm})$ & Measurement 2 accuracy error $(\mathrm{mm})$ \\
\hline Form 2 & $15 \mathrm{~h} 20 \mathrm{~m} \pm 2 \mathrm{~h} \mathrm{07} \mathrm{m}$ & $\$ 33.64 \pm \$ 5.52$ & $0.44 \pm 0.48$ & $0.30 \pm 0.12$ \\
Objet & $20 \mathrm{~h} 03 \mathrm{~m} \pm 2 \mathrm{~h} 23 \mathrm{~m}$ & $\$ 107.70 \pm \$ 9.82$ & $0.28 \pm 0.35$ & $0.04 \pm 0.09$ \\
Ultimaker & $16 \mathrm{~h} 40 \mathrm{~m} \pm 3 \mathrm{~h} 23 \mathrm{~m}$ & $\$ 4.00 \pm \$ 0.69$ & $0.82 \pm 0.47$ & $0.34 \pm 0.17$ \\
uPrint & $29 \mathrm{~h} 04 \mathrm{~m} \pm 3 \mathrm{~h} 56 \mathrm{~m}$ & $\$ 43.04 \pm \$ 6.80$ & $0.30 \pm 0.12$ & $0.30 \pm 0.12$ \\
\hline
\end{tabular}

Print times are as reported by the printer. Costs are calculated in USD (\$). Measurement 1 Accuracy Error is the mean absolute error between the posterior inferior iliac spine and the ischial spine (as measured in $\mathrm{mm}$ ) and Measurement 2 Accuracy Error is the mean absolute error for the fracture fragment (as measured in $\mathrm{mm}$ ).

FDM printer without dissolvable supports. An outline of the segmentation software and printers used can be found in Figure 1.

\section{Model price}

The price to produce each model was based solely on the amount of material used and did not include engineering time, since roughly each model took approximately 60 minutes to develop (segmentation time). The price for each uPrint (high-end FDM) model was $\$ 43.04 \pm 6.80$ and $\$ 33.64 \pm 5.52$ for each Form 2 (low-cost resin) model. The price per Objet (high-end resin) model was significantly higher at $\$ 107.70 \pm 9.82$, and the price of the Ultimaker (low-cost FDM) model was significantly lower at $\$ 4.00 \pm 0.69$ per model. Additional information regarding printing time can be found on Table 1 .

\section{Model evaluation}

The $3 \mathrm{D}$ printed models were evaluated based on their accuracy as well as their utility in displaying the clinically significant information. In order to measure model accuracy in displaying the region of interest, digital measurements were taken on the original CT imaging data using the Change picture archiving and communication system (PACS) (Change Healthcare, USA) of the distance between specific landmarks and then replicated on the physical 3D printed models. Measurements were performed on multiplanar maximum intensity projection (MIP) $10 \mathrm{~mm}$ reformats, which were sufficient to identify the landmarks of interest. The distance between the posterior inferior iliac spine (PIIS) and the ischial spine was measured across all models as an easily identifiable anatomical landmark as a way to quantitatively assess accuracy of the representation (Figure 2). In addition, an easily identifiable bone fragment within the fracture was measured for each case as a pathological measurement. First, digital measurements were taken using Change PACS. The measurements were then repeated on the $3 \mathrm{D}$ renderings after segmentation using the respective segmentation software. Then, separately, physical measurements taken from the $3 \mathrm{D}$ printed models were recorded using high-grade digital calipers (PEC Tools, Santa Monica, CA, USA) by a second observer who was blinded to the model type (15). Measurements were rounded to the nearest tenth of a millimeter on both the digital measurements and the physical measurements. The difference between the measurement recorded on the CT scan and the physical 3D printed model was recorded and used for data analysis as the measurement error; absolute values were also recorded to analyze errors without direction bias.

\section{Clinical evaluation}

The models were evaluated using a 15-question survey (10 point Likert scale, with 10 being a positive response, and 1 being a negative response) adapted from previous observations of anatomical modeling within surgical planning $(11,16)$. Five physicians (one orthopedic surgeon involved in the operation, three radiologists, and an anesthesiologist) provided clinical feedback about the models. Questions include topics such as accuracy, production time, assistance in planning, efficiency, inventory management, potential complication avoidance, recommendation for future operations (Appendix 1).

\section{Statistical analysis}

The material costs associated with manufacturing were calculated based on the amount of material used and cost per weight of the material. Two one-way ANOVAs were 

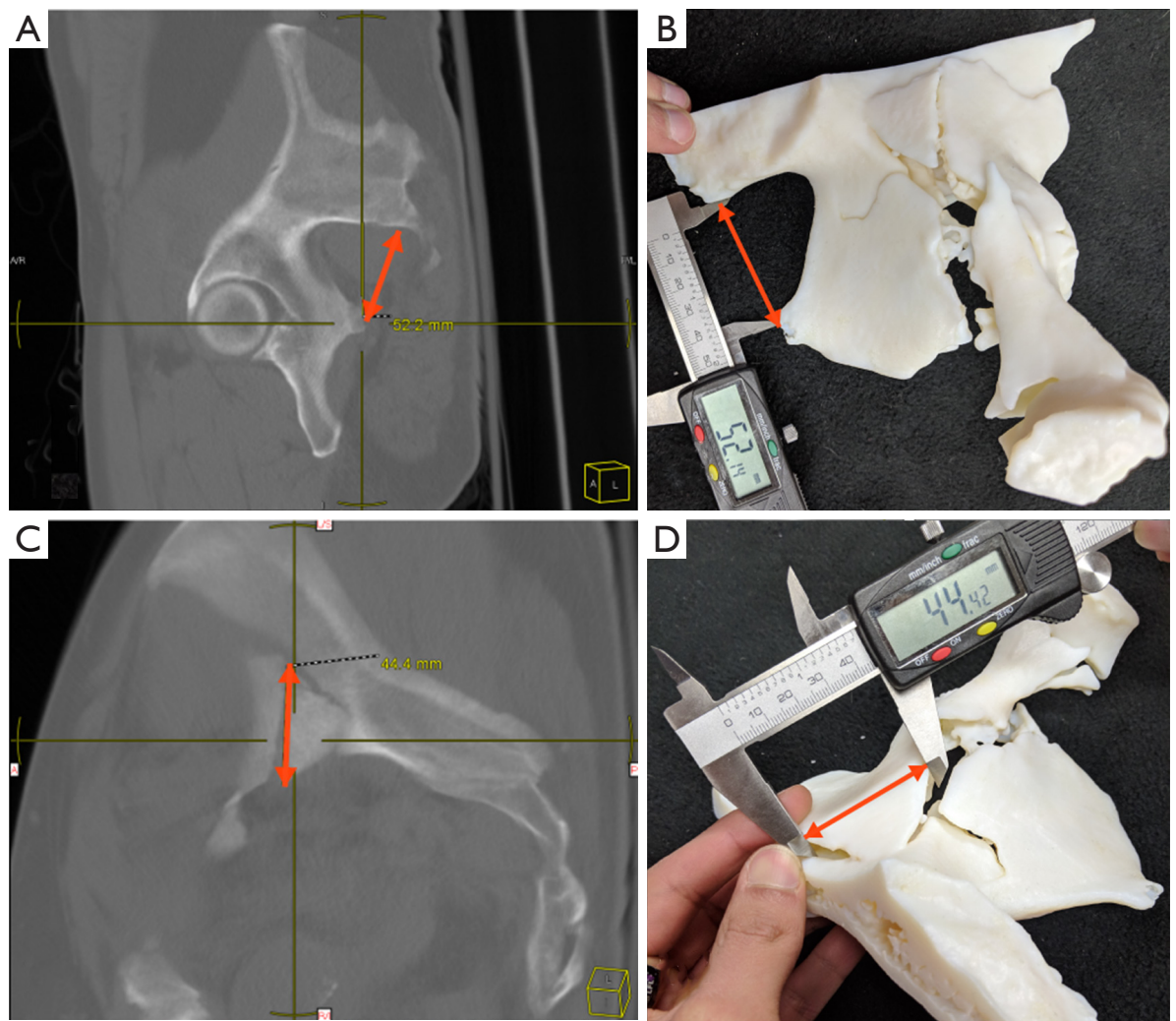

Figure 2 Example of the measurements taken to observe the distance between the posterior inferior iliac spine (PIIS) and the ischial spine on (A) the computer tomography (CT) data and (B) the 3D printed model, as well as the unique fracture fragment on (C) the CT data and (D) the $3 \mathrm{D}$ printed model.

used to analyze the mean absolute error values for the distance recorded between the PIIS and the ischial spine as well as the error values of the fracture fragment for each of the 4 printers used with a significance level of $\mathrm{P} \leq 0.05$. A one-way ANOVA was used to compare the survey results of the different models produced, with a significance level of $\mathrm{P} \leq 0.05$.

In addition, the data was also analyzed using a BlandAltman analysis $(17,18)$. Pearson product-moment correlation coefficient was calculated to examine the correlations between the difference and the mean of the difference from the mean values and explain the agreement between the two quantitative measures taken. A $\mathrm{P}$ value $\leq 0.05$ was considered statistically significant for the measurement of proportional bias.

\section{Results}

\section{Model descriptions}

The following measurements were recorded when measuring the distance from the PIIS to the ischial spine directly on the CT images: Model 1 at $47.0 \mathrm{~mm}$, Model 2 at $53.7 \mathrm{~mm}$, Model 3 at $51.5 \mathrm{~mm}$, Model 4 at $52.5 \mathrm{~mm}$, and Model 5 at $70.0 \mathrm{~mm}$.

The fracture fragments used to measure the precision of the printing method varied depending on the nature of the fracture and was measured directly on the CT imaging data. Model 1 was a both-column acetabular fracture, and the measured fragment was posterior and superior to the acetabulum and measured $63.2 \mathrm{~mm}$. Model 2 was an acetabular fracture with a protrusion, with the fracture fragment located anterior to the acetabulum on the superior aspect measuring $35.4 \mathrm{~mm}$. Model 3 was a gunshot acetabular fracture with the measured fragment posterior to the acetabulum and measuring $41.4 \mathrm{~mm}$. Model 4 was a both column acetabular fracture with the measured fragment being a triangular fragment along the inner iliac wing and measured $44.4 \mathrm{~mm}$. Model 5 was a posterior acetabular fracture with the fragment being superior to the hip joint and measured $38.1 \mathrm{~mm}$. 
Table 2 Measurements of the distance between the posterior inferior iliac spine (PIIS) and the ischial spine (IS) and the unique acetabular fracture fragment for models segmented on 3D Slicer and Mimics

\begin{tabular}{|c|c|c|c|c|c|c|}
\hline Model & \multicolumn{2}{|c|}{ Original CT (mm) } & \multicolumn{2}{|c|}{ 3D Slicer Segmentation $(\mathrm{mm})$} & \multicolumn{2}{|c|}{ Mimics Segmentation (mm) } \\
\hline 1 & 47.0 & 63.2 & 47.6 & 62.9 & 47.3 & 62.4 \\
\hline 2 & 53.7 & 35.4 & 53.4 & 34.6 & 53.6 & 34.8 \\
\hline 3 & 51.5 & 41.4 & 52.0 & 40.7 & 52.2 & 41.4 \\
\hline 5 & 70.0 & 38.1 & 69.1 & 37.7 & 69.6 & 38.0 \\
\hline
\end{tabular}

A

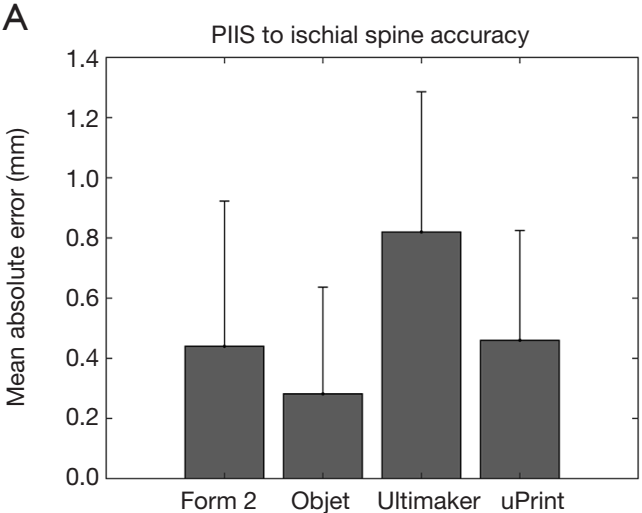

B

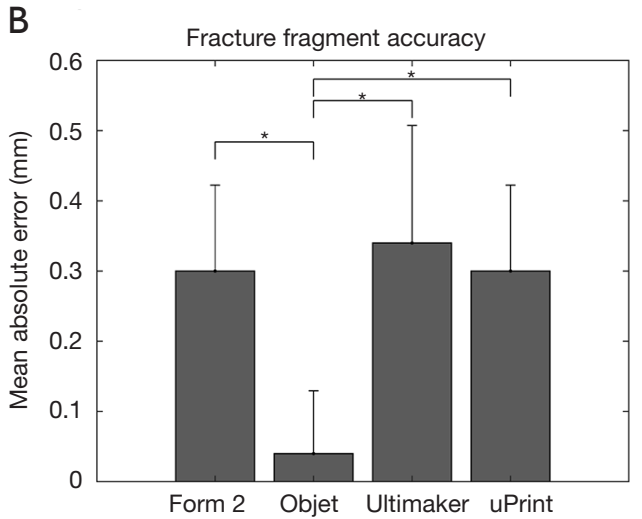

Figure 3 Average absolute value measurement error of (A) the distance between the posterior inferior iliac spine (PIIS) and the ischial spine and (B) the acetabular fracture fragment. No significant differences were found between the distance from the PIIS to the ischial spine. There was a significant difference between the measurement of the fracture fragment on the models printed by the Objet compared to the uPrint $(\mathrm{P}=0.026)$, Form $2(\mathrm{P}=0.026)$, and Ultimaker $(\mathrm{P}=0.010) .{ }^{*} \mathrm{P}<0.05$.

Measurements of the distance between the PIIS to the ischial spine and the acetabular fracture fragment were assessed with the respective software that was used for the segmentation process and can be seen in Table 2. The mean absolute error from the software measurements was found not to be statistically significant between software types for both the PIIS to ischial spine distance and the fracture fragment distance, $\mathrm{F}(3,19)=0.426, \mathrm{P}=0.737$.

\section{Measurement comparison}

The one-way ANOVA showed that there was no significant difference in the measurement error between the models produced from either method, $\mathrm{F}(3,19)=1.459, \mathrm{P}=0.263$ (Figure $3 A$ ). When comparing the error in the fracture fragment measurement between the models, there was a significant difference between the Objet and the other three methods, $\mathrm{F}(3,19)=5.768, \mathrm{P}=0.007$. The Objet models had significantly less error than the uPrint models $(\mathrm{P}=0.026)$, Form 2 models $(\mathrm{P}=0.026)$, and the Ultimaker models $(\mathrm{P}=0.010)$ (Figure $3 B)$. Examination of the distributions of measurement errors indicate physical models both over- and under-estimated the true distance with a slight bias towards over-estimation on all four printers (Figure 4).

The results of the Bland-Altman analysis show that the differences between production methods resulted in similar results when comparing the representation of the general anatomy as well as the fracture fragment. In each BlandAltman plot produced, a majority of the values fell within two standard deviations of the mean. For all the BlandAltman analyses, there were no significant linear regression values for the PIIS to ischial spine measurement or the fracture measurement $(r=0.022$ and $r=0.103$, respectively) or indications of proportional bias $(\mathrm{P}=0.927$ and $\mathrm{P}=0.625$, 

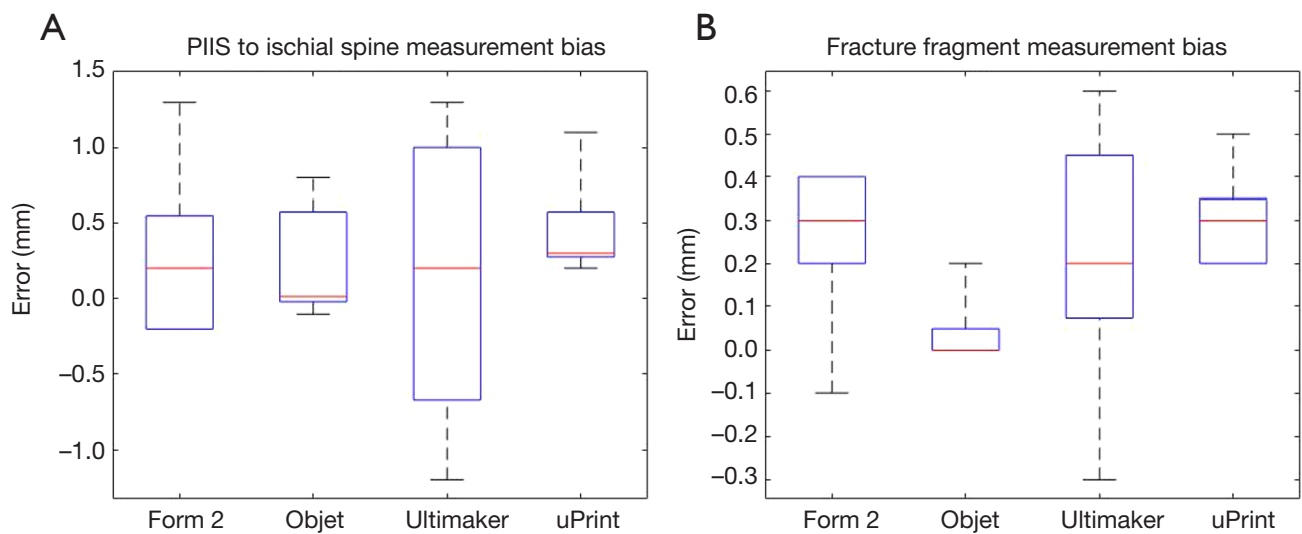

Figure 4 Boxplot of the raw measurement mean error for (A) the distance between the posterior inferior iliac spine (PIIS) and the ischial spine and (B) the acetabular fracture fragment.
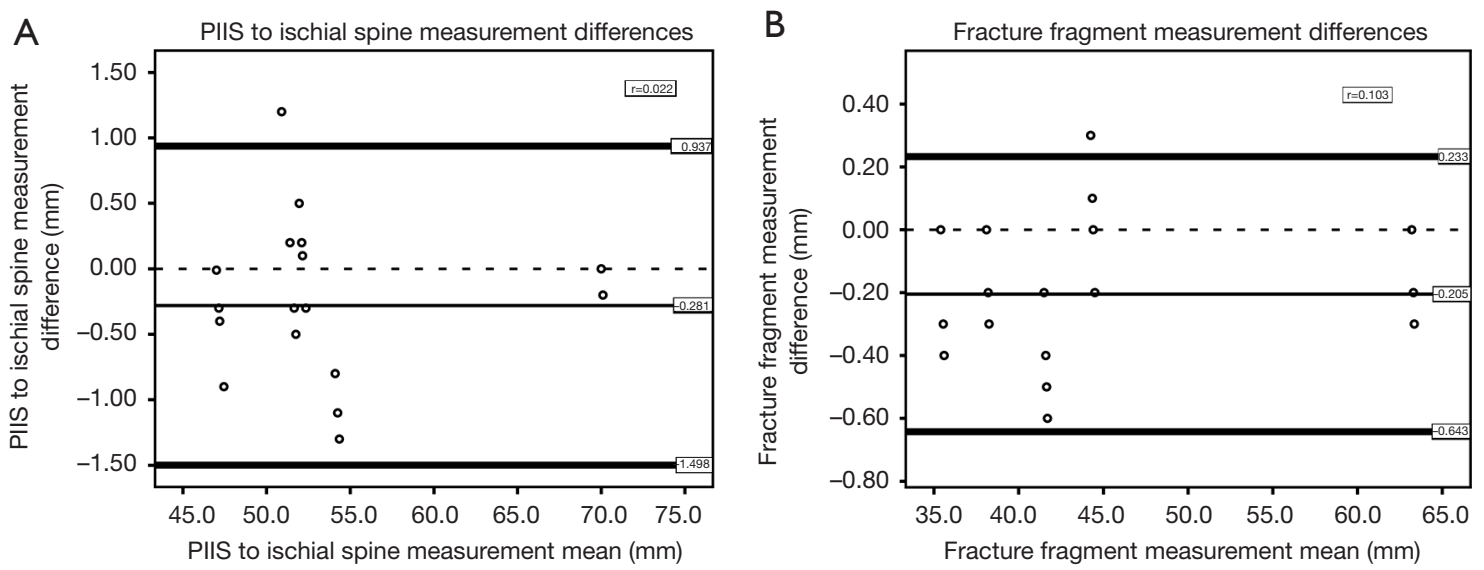

Figure 5 Bland-Altman analysis of the measurement error of (A) the distance between the posterior inferior iliac spine (PIIS) and the ischial spine and (B) the acetabular fracture fragment. For both the PIIS to the ischial spine measurement and the fracture measurement, there were no significant linear regression values $(r=0.022$ and $r=0.103$, respectively) or indications of proportional bias $(\mathrm{P}=0.927$ and $\mathrm{P}=0.625$, respectively).

respectively) (Figure 5).

\section{Clinical survey comparison}

For the survey analysis, only questions that were relevant to the study and received more than one response were included in the analysis (Table 3). This meant the exclusion of Question 5, Question 8, Question 9, Question 10, Question 11, and Question 12. These questions were outside the scope of the study and/or did not receive sufficient feedback $(\mathrm{N} \leq 1)$.

The one-way ANOVA of the survey data indicated that there was a significant difference between model types $\mathrm{F}(3,79)=4.256, \mathrm{P}=0.008$. Tukey post-hoc analysis showed that there was a significant difference in the perceived representation of the fracture between the uPrint (highend FDM) and the Ultimaker (low-cost FDM) $(\mathrm{P}=0.047)$, as well as the Objet (high-end resin) and Ultimaker (lowcost $\mathrm{FDM})(\mathrm{P}=0.032)$. There were no significant differences found between the other questions and model types included in the survey. The low sample size of the survey and number of responses indicate the survey is subject to potential bias and will be addressed more thoroughly in the discussion. 


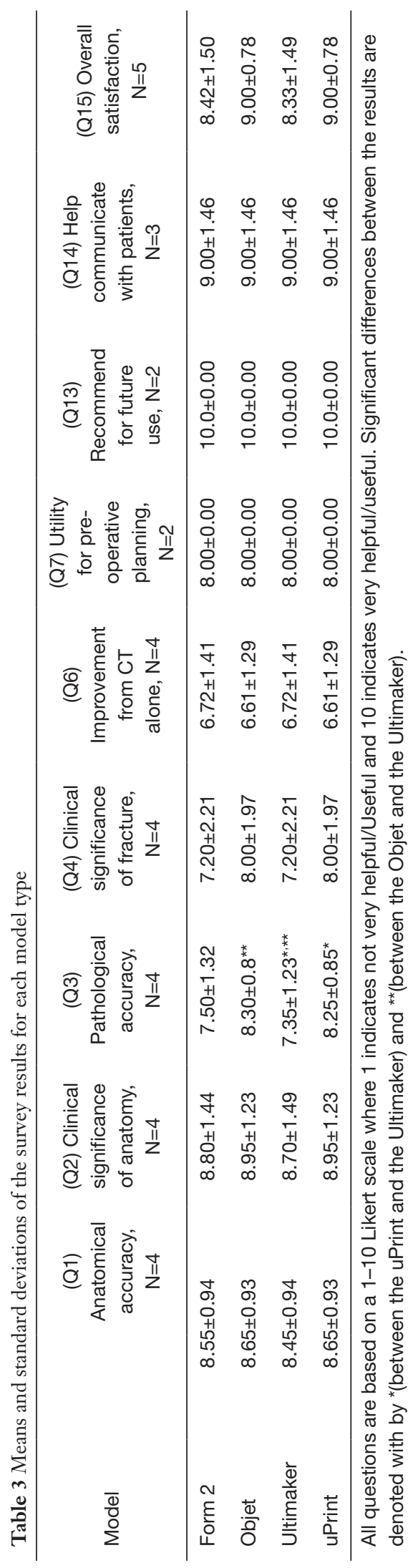

\section{Discussion}

The results of this study found that the type of printer used to develop acetabular fracture models has a slight effect on the final product. When analyzing the mean absolute error of the distance between the PIIS and the ischial spine compared to the original CT, there were no significant differences between any of the models produced $(\mathrm{P}=0.263)$. However, the high-end resin (Objet) printer had a significantly different mean absolute error of the fracture fragment measurement than the other printers $(\mathrm{P}=0.007)$ indicating higher accuracy, which was expected. Of the 9 different survey questions used for analysis, there was only a significantly lower difference in the representation of the pathology when using the low-cost FDM method $(7.35 \pm 1.23)$ when compared to the two different high-end prints $(\mathrm{FDM}=8.25 \pm 0.85$, Resin $=8.30 \pm 0.80)$.

The purpose of this study was to observe if acetabular fracture models could be produced using more affordable methods while still maintaining the same clinical validity as high-end models. Comparisons made between the pathological measurements indicated that the models produced by the high-end resin (Objet) printer were significantly more precise than the models produced by all 3 other printers $(\mathrm{P}=0.007)$. In this case, the error was $0.3 \mathrm{~mm}$, which was only $0.48 \%$ of the largest fracture length $(63.2 \mathrm{~mm})$ and $0.85 \%$ of the smallest fracture length $(35.4 \mathrm{~mm})$, and thus unlikely to be clinically significant. While this small error may be negligible in structures as large as the acetabulum, it could prove significant when modeling small structures or vasculature in pediatric patients. Furthermore, this small difference in the fracture pattern was also reflected by the physicians who provided feedback on the anatomic models. There was a significant difference in Question 3, which asked "Is the model an accurate representation of the pathology?" $(\mathrm{P}=0.047)$. However, the subsequent question in the survey asked "If there are inaccuracies to the pathology representation, are they clinically significant to you?" and this was found not to be significant $(\mathrm{P}=0.412)$. This suggests that the model differences produced by each method (both high-end and low-cost) are not significant enough to result in a different clinical outcome, or that they still convey the same amount of information about the fracture and can still be utilized for things such as surgical planning. Currently, 3D Slicer is intended for research purposes, rather than clinical application like Mimics is. However, the results of this study indicate that the open-source software could offer some utility to institutions that do not have the resources for 
proprietary software.

The results of the survey suggest that anatomical models can play a valuable role in pre-surgical planning regardless of the methodology used for development. All of the acetabular models were found to be accurate representations of the general anatomy (average of $8.58 \pm 0.94$ ), and that any inaccuracies between the imaging data and the model itself were not clinically relevant for both the general anatomy $(8.85 \pm 1.35)$ and for the fracture itself (7.6 2.09). Additionally, the 3D model provides a new and useful perspective compared to $2 \mathrm{D}$ CT images alone $(6.67 \pm 1.35)$ and that there was an overall satisfaction with the final product $(8.69 \pm 1.14)$. However, one major limitation from this study is the quality of information that can be drawn from the survey portion. The models were reviewed by 5 participants in different fields of medicine, not all of which would use the model for the intended surgical planning purpose and may be subject to a fair amount of bias. The survey suggests that the models are representative and accurate, however may not be conclusive from this study alone.

Cost has often been cited as a barrier to entry for $3 \mathrm{D}$ printing, which is especially true for developing countries (19). Any usage after the initial purchase is reduced to only the cost of the material, which for this experiment ranged from $\$ 3.30$ to $\$ 4.70$ for the low-cost FDM models. Many of the questions included on the survey indicate that the models produced are both accurate representations of the overall structure (average score of 8.58 ) and a useful step in planning the operation (average score of 8). This indicates that for as low as $\$ 3.30$, it is possible to create an acetabular model that could both improve surgery preparation as well as teach students about complex cases that may arise. In one educational application, a model developed for $\sim \$ 10$ was estimated to have saved 45 minutes within the operating room, and was equivalent to saving $\$ 2,700$ for the hospital (8). While these numbers are estimates, they help to show how the monetary benefits from this type of education would be difficult to calculate but likely outweigh the cost of the material for facilities with less access to resources.

While some hospitals are well funded and can afford to use equipment regardless of its cost for medical $3 \mathrm{D}$ printing, hospitals with less abundant resources likely do not have this option. This study was designed to test the entire production method from segmentation to final product with this application in mind. A portion of error was included as a result of both segmentation methods, however the difference between them was not significant. This suggests that the main source of difference between the final product is the printer used for manufacturing. Based on the hardware limits of the $3 \mathrm{D}$ printers, it has been reported that models produced using SLA methods/resin based material would likely have the most accurate results in reflecting the anatomy (20). However, the error differences found in this study were extremely small, often times less than $1 \mathrm{~mm}$, and would likely not play a significant role in surgical planning for orthopedic purposes. Even with the slight differences in accuracy, the low-cost FDM model had similar physician feedback like what has been reported in other studies, and should encourage its usage in more countries $(21,22)$.

Focusing specifically on the acetabulum for model development allows for easier comparison between production types, however, makes it more difficult to apply the results to models of different structures such as the heart or the liver for complex tumor representations. 3D printing specifically for orthopedics will likely have similar results, but more complex multi-part models may need access to more material textures and colors. This type of material mixing currently does not easily exist for lowcost 3D printing. High-end printers like the Objet that are capable of mixing materials have much more versatility in what types of models can be produced. The results of this study only reflect single material anatomical models but could be replicated with a low-cost multi-material printer once it becomes more accessible.

As previous research has indicated, the utility of $3 \mathrm{D}$ printed models is only beginning to be explored due to the number of factors that can influence model utilization. This includes the purpose of the model, imaging techniques used to drive segmentation, segmentation expertise and software used, and printer specifications (9). In some cases, information such as operating room time and follow-up time were recorded to observe the impact of the models $(8,23,24)$. However, a majority of the articles involving anatomical modeling report little to no qualitative information (25-28). One reason for the large variety of measured outcomes is that different operations will have different measures of success. The differences in model purpose make comparing the effectiveness of each model unique to its application, and the results from one study may not be generalizable to other studies.

These findings indicate that low-cost methods of developing 3D printed acetabular fracture and possibly other models may be sufficient to produce accurate representations that provide substantial clinical value to the surgeons. Therefore, the practice of printing models 
to improve surgical outcomes should be feasible for clinics with limited funding. Future studies should determine the extent to which the low-cost application is feasible through implementation in different regions of the body as well as impacts on different operations outcomes and which ones are relevant to their study.

\section{Conclusions}

This study observed 3D printed acetabular fracture models made using different production methods, their representations of the fracture, and potential utility as a method of surgical planning. The results found that while there was a minor difference in the accuracy between the methods of production, there was no difference between the quality of information that the models provided. Hospitals with less access to resources could potentially use this information to consider including low-cost $3 \mathrm{D}$ printed anatomic models for their complex surgical procedures.

\section{Acknowledgments}

We would like to thank Dr. Ka-Chun "Joseph" Siu (University of Nebraska Medical Center) for his feedback on the study.

Funding: This research was partially funded by the National Institute of Neurological Disorders and Stroke of the National Institutes of Health under Award Number R01NS114282 and the Biomechanical Rehabilitation and Manufacturing Initiative (BRMI), as well as the NASA Nebraska Space Grant.

\section{Footnote}

Reporting Checklist: The authors have completed the MDAR reporting checklist. Available at https://atm.amegroups. com/article/view/10.21037/atm-21-5069/rc

Data Sharing Statement: Available at https://atm.amegroups. com/article/view/10.21037/atm-21-5069/dss

Peer Review File: Available at https://atm.amegroups.com/ article/view/10.21037/atm-21-5069/prf

Conflicts of Interest: All authors have completed the ICMJE uniform disclosure form (available at https://atm. amegroups.com/article/view/10.21037/atm-21-5069/coif). The authors have no conflicts of interest to declare.
Ethical Statement: The authors are accountable for all aspects of the work in ensuring that questions related to the accuracy or integrity of any part of the work are appropriately investigated and resolved. The study was conducted in accordance with the Declaration of Helsinki (as revised in 2013). This study was approved through the IRB at the University of Nebraska Medical Center (258-18-EP) with waiver of consent due to the data being deidentified and accessed retrospectively

Open Access Statement: This is an Open Access article distributed in accordance with the Creative Commons Attribution-NonCommercial-NoDerivs 4.0 International License (CC BY-NC-ND 4.0), which permits the noncommercial replication and distribution of the article with the strict proviso that no changes or edits are made and the original work is properly cited (including links to both the formal publication through the relevant DOI and the license). See: https://creativecommons.org/licenses/by-nc-nd/4.0/.

\section{References}

1. Rinne PP, Laitinen MK, Kannus P, et al. The incidence of pelvic fractures and related surgery in the Finnish adult population: a nationwide study of 33,469 patients between 1997 and 2014. Acta Orthop 2020;91:587-92.

2. Mauffrey C, Hao J, Cuellar DO 3rd, et al. The epidemiology and injury patterns of acetabular fractures: are the USA and China comparable? Clin Orthop Relat Res 2014;472:3332-7.

3. Boudissa M, Francony F, Kerschbaumer G, et al. Epidemiology and treatment of acetabular fractures in a level-1 trauma centre: Retrospective study of 414 patients over 10 years. Orthop Traumatol Surg Res 2017;103:335-9.

4. Whiting PS, Anderson DR, Galat DD, et al. State of Pelvic and Acetabular Surgery in the Developing World: A Global Survey of Orthopaedic Surgeons at Surgical Implant Generation Network (SIGN) Hospitals. J Orthop Trauma 2017;31:e217-23.

5. Mesbahi SAR, Ghaemmaghami A, Ghaemmaghami S, et al. Outcome after Surgical Management of Acetabular Fractures: A 7-Year Experience. Bull Emerg Trauma 2018;6:37-44.

6. Jacobs S, Grunert R, Mohr FW, et al. 3D-Imaging of cardiac structures using 3D heart models for planning in heart surgery: a preliminary study. Interact Cardiovasc Thorac Surg 2008;7:6-9. 
7. Salazar D, Huff TJ, Cramer J, et al. Use of a threedimensional printed anatomical model for tumor management in a pediatric patient. SAGE Open Med Case Rep 2020;8:2050313X20927600.

8. Cherkasskiy L, Caffrey JP, Szewczyk AF, et al. Patientspecific 3D models aid planning for triplane proximal femoral osteotomy in slipped capital femoral epiphysis. J Child Orthop 2017;11:147-53.

9. Mitsouras D, Liacouras P, Imanzadeh A, et al. Medical 3D Printing for the Radiologist. Radiographics 2015;35:1965-88.

10. Zheng YX, Yu DF, Zhao JG, et al. 3D Printout Models vs. 3D-Rendered Images: Which Is Better for Preoperative Planning? J Surg Educ 2016;73:518-23.

11. Birbara NS, Otton JM, Pather N. 3D Modelling and Printing Technology to Produce Patient-Specific 3D Models. Heart Lung Circ 2019;28:302-13.

12. Martin CM, Roach VA, Nguyen N, et al. Comparison of $3 \mathrm{D}$ reconstructive technologies used for morphometric research and the translation of knowledge using a decision matrix. Anat Sci Educ 2013;6:393-403.

13. Ripley B, Levin D, Kelil T, et al. 3D printing from MRI Data: Harnessing strengths and minimizing weaknesses. J Magn Reson Imaging 2017;45:635-45.

14. Fedorov A, Beichel R, Kalpathy-Cramer J, et al. 3D Slicer as an image computing platform for the Quantitative Imaging Network. Magn Reson Imaging 2012;30:1323-41.

15. Leng S, McGee K, Morris J, et al. Anatomic modeling using 3D printing: quality assurance and optimization. 3D Print Med 2017;3:6.

16. Bagaria V, Chaudhary K. A paradigm shift in surgical planning and simulation using 3Dgraphy: Experience of first 50 surgeries done using 3D-printed biomodels. Injury 2017;48:2501-8.

17. Zuniga J, Katsavelis D, Peck J, et al. Cyborg beast: a lowcost 3d-printed prosthetic hand for children with upperlimb differences. BMC Res Notes 2015;8:10.

18. Bland JM, Altman DG. Statistical methods for assessing

Cite this article as: Salazar DA, Cramer J, Markin NW, Hunt NH, Linke G, Siebler J, Zuniga J. Comparison of 3D printed anatomical model qualities in acetabular fracture representation. Ann Transl Med 2022;10(7):391. doi: 10.21037/atm-21-5069 agreement between two methods of clinical measurement. Lancet 1986;1:307-10.

19. Tetsworth KD, Mettyas T. Overview of Emerging Technology in Orthopedic Surgery. Tech Orthop 2016;31:143-52.

20. AlAli AB, Griffin MF, Butler PE. Three-Dimensional Printing Surgical Applications. Eplasty 2015;15:e37.

21. Mottl-Link S, Hübler M, Kühne T, et al. Physical models aiding in complex congenital heart surgery. Ann Thorac Surg 2008;86:273-7.

22. Velasco I, Vahdani S, Ramos H. Low-cost Method for Obtaining Medical Rapid Prototyping Using Desktop 3D printing: A Novel Technique for Mandibular Reconstruction Planning. J Clin Exp Dent 2017;9:e1103-8.

23. Zheng W, Tao Z, Lou Y, et al. Comparison of the Conventional Surgery and the Surgery Assisted by $3 \mathrm{~d}$ Printing Technology in the Treatment of Calcaneal Fractures. J Invest Surg 2018;31:557-67.

24. Martelli N, Serrano C, van den Brink H, et al. Advantages and disadvantages of 3-dimensional printing in surgery: A systematic review. Surgery 2016;159:1485-500.

25. Anderson JR, Thompson WL, Alkattan AK, et al. Threedimensional printing of anatomically accurate, patient specific intracranial aneurysm models. J Neurointerv Surg 2016;8:517-20.

26. Xie M, Tang K, Yuan C. 3D printing lunate prosthesis for stage IIIc Kienböck's disease: a case report. Arch Orthop Trauma Surg 2018;138:447-51.

27. Igami T, Nakamura Y, Hirose T, et al. Application of a three-dimensional print of a liver in hepatectomy for small tumors invisible by intraoperative ultrasonography: preliminary experience. World J Surg 2014;38:3163-6.

28. Schmauss D, Haeberle S, Hagl C, et al. Threedimensional printing in cardiac surgery and interventional cardiology: a single-centre experience. Eur J Cardiothorac Surg 2015;47:1044-52. 


\section{Supplementary}

\section{Questions asked for Clinical Evaluation:}

1. Is the model an accurate representation of the anatomy?

2. If there are inaccuracies to the anatomical representation, how clinically significant are they to you?

3. Is the model an accurate representation of the pathology?

4. If there are inaccuracies to the pathology representation, are they clinically significant to you?

5. Was the time taken to produce the model satisfactory?

6. Did the model provide a different perspective from $x$-ray/CT alone?

7. Would the model be helpful in preoperative planning?

8. Did seeing the model alter your initial surgical plan that was based on radiographs and CT?

9. Do you believe it would help in reducing surgical time?

10. Would it improve the inventory management?

11. Would the model help avoid any surgical complications?

12. Did the post-operative $\mathrm{x}$-ray prove to be satisfactory and in accordance with your intraoperative expectations?

13. Would you recommend the use of anatomical models for cases in the future?

14 . Would the $3 \mathrm{D}$ printed model help you communicate with patients?

15. Overall satisfaction with the $3 \mathrm{D}$ printed model? 